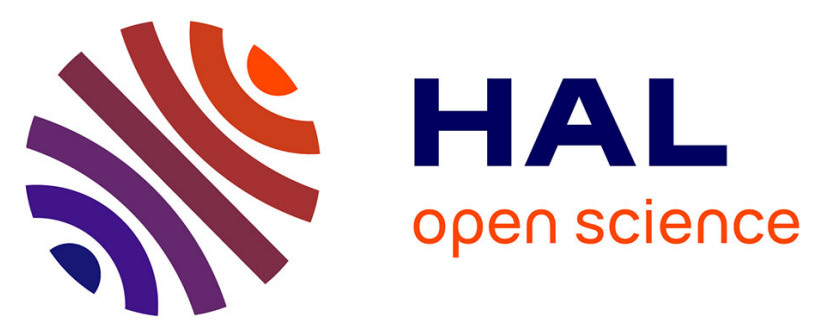

\title{
On inverse scattering and imaging solutions for objects buried within uniaxially anisotropic media
}

Dominique Lesselier, Ping-Ping Ding, Giacomo Rodeghiero, Marc Lambert, Yu Zhong

\section{- To cite this version:}

Dominique Lesselier, Ping-Ping Ding, Giacomo Rodeghiero, Marc Lambert, Yu Zhong. On inverse scattering and imaging solutions for objects buried within uniaxially anisotropic media. IEEE Conference - 15th IEEE Mediterranean Microwave Symposium, Nov 2015, Lecce, Italy. pp.ID-16, 10.1109/MMS.2015.7375424 . hal-01236175

\section{HAL Id: hal-01236175}

https://hal-centralesupelec.archives-ouvertes.fr/hal-01236175

Submitted on 1 Dec 2015

HAL is a multi-disciplinary open access archive for the deposit and dissemination of scientific research documents, whether they are published or not. The documents may come from teaching and research institutions in France or abroad, or from public or private research centers.
L'archive ouverte pluridisciplinaire HAL, est destinée au dépôt et à la diffusion de documents scientifiques de niveau recherche, publiés ou non, émanant des établissements d'enseignement et de recherche français ou étrangers, des laboratoires publics ou privés. 


\title{
On inverse scattering and imaging solutions for objects buried within uniaxially anisotropic media
}

\author{
Dominique Lesselier, Ping-Ping Ding, \\ Giacomo Rodeghiero \\ Laboratoire des Signaux et Systèmes \\ CNRS-CentraleSupélec-Univ. Paris Sud \\ 3, rue Joliot Curie, 91192 Gif-sur-Yvette, France \\ givenname.name@12s.centralesupelec.fr
}

\author{
Marc Lambert \\ Génie Electrique and Electronique de Paris \\ CNRS-CentraleSupélec-Univ. Paris Sud-Univ. Pierre et \\ Marie Curie \\ 11, rue Joliot Curie, 91192 Gif-sur-Yvette, France \\ givenname.name@geeps.centralesupelec.fr
}

\author{
Yu Zhong \\ A*STAR, Institute of High Performance Computing \\ 138632 Singapore. \\ zhongyu@ihpc.a-star.edu.sg
}

\begin{abstract}
Electromagnetic imaging of damaged uniaxially anisotropic composite materials certainly remains a challenging task yet it is in need for whatever concerns quality, viability, safety and availability of systems involving manufactured composite parts, e.g., in aeronautics and in automotive industry. The contribution first surveys in some depth the investigation topic with examples being drawn from the most recent literature by the authors and others. Then, upon introduction of a rigorous full-wave 3-D electromagnetic modeling and asymptotic firstorder counterpart, retrieval of volumetric defects hidden in such materials via MUSIC approaches (under various guises), in the case of defects small enough, and Subspace Optimization Methods, for larger ones, will be exhibited from single-frequency multi-static data acquired in the reflection mode.
\end{abstract}

Keywords-electromagnetics; scattering; uniaxial anisotropy; inversion; imaging; MUSIC; RAP-MUSIC; SOM

\section{INTRODUCTION}

Focus is on microwave imaging of complex dispersive, possibly anisotropic artificial structures that are amenable to such an investigation. Good but not only examples are damaged fiber-based composite structures encountered in aeronautic and automotive industries. Many imaging methods are around, relying on various linearized and non-linearized representations of wave phenomena, with different degrees of reliability and faithfulness to the physics. Intertwined questions are on retrievable information, regularization and priors (e.g., sparsity), confidence levels, in tune with endusers' needs. This makes a strong, multi-pronged challenge.

Here one is considering contrast-source-based, reliable imaging of their 3-D damages, emphasizing ways to get leastcomputation-cost results, account for proper priors, via parallel/synergized/ studies on smart, feature-rich Subspace Optimization Methods (SOM) and quick/one-shot or a-fewiterations-involved MUSIC (MUltiple SIgnal Classification) methods, under various guises. Bayesian Compressive Sensing methods and L1-minimizing sparsity-promoting approaches in a deterministic framework apply as well, but are left aside at this time in the numerical illustrations at least.

The contribution has two purposes, to introduce the investigation topic with the help of a good number of relevant publications, and to point out pros and cons of aforementioned SOM and MUSIC methods via two illustrative results on a common 3-D anisotropic configuration. No attempt is made to go into the mathematical and computational subtleties, referring the reader to the authors' most recent contributions in which the above issues are considered in depth.

\section{Modeling AND IMAGING AT A GLANCE}

\section{A. About large-scale and small-scale models of composites}

It is easy to observe that most of the artificial materials in use in either recent realizations in the aeronautic industry (concerning both civil and military airplanes marketed already) or in recent developments in the automotive industry (mostly at concept-car level so far) that are really accessible to microwave imaging are fibrous composites, once noticed that many other cases are of interest as well, paintings, covers, etc. Refer to [1] as an excellent source, and a rather replete one, and in view of testing of carbon-fiber-reinforced structures to the exhaustive and promising analysis in [2].

Be a panel structure: it can be considered as a pile of planar plates (at least since sources and sensors view only a limited portion of the structure under test, with almost nil curvature, locally speaking), one plate lying over the other, the panel itself being sandwiched between a cover (usually an air half-space) and a substrate (air half-space, metal sheet); each plate consists of a regular arrangement of long cylinders (long enough again vs. the extent of the illumination/ observation) with similar finite (circular as being the option here) sections, 
all such cylinders being orientated into the same direction and their constitutive material differing from the embedding material (matrix) which they are reinforcing.

Two models follow. At large scale (large enough local wavelength vs. main geometric features), locally averaged tensor electromagnetic parameters inserted into Maxwell's PDE of concern are assumed for each plate. At small scale (small enough local wavelength vs. geometry), each plate in the panel is taken periodic, with given pattern within an elementary subdivision (cell) repeated in the other cells into a direction parallel with the planar interfaces. In any plate each cell should contain a circular cylinder (fiber bundle) with due repetition, the common orientation changing from plate to plate; concurrent methods then appear, depending on seeing each plate as infinite array (prone to Floquet modeling, e.g., [3] and references therein) or finite one.

In both hypotheses, damages of various kinds matter: at large scale, thin cracks traversing one or more plates, volumetric, bubble-like inclusions, and plate delamination; at small scale, missing, misplaced or disorientated cylinders or sets of cylinders. And one has to simulate the electromagnetic response both of an undamaged structure and of a damaged one, as unavoidable step along development/simulation/ of any well-conceived imaging procedure.

Let us note that in microwaves, what is often done in NdT practice is to move waveguide applicators close to the panel's interfaces and measure reflection and/or transmission coefficients whose variations should thus be typical of defects in the zone probed [4-5], and again [1-2] and references therein, special $\mathrm{THz}$ cases (or better said, equivalent ones in the transient time-domain) appearing as well as of lately, e.g., in military aeronautics [6].

Subspace-based Optimization Methods (SOM) [7-9] have never been applied in the present context. Yet they appear to converge fast and robustly vs. strong measurement noise, and to be computation-efficient by decreasing the non-linearity of the inversion while (if smartly implemented) of manageably low computational cost in the standard free-space configurations that are the only ones seemingly investigated so far (2-D, lately, 3-D). An example of a recent development by the authors for the large-scale anisotropic model is given next.

Attempting a quick description of SOM in a Green-based contrast source formulation (managed via Methods of Moments), the induced-current-to-field mapping operator is decomposed so as induced currents in the Region of Interest (RoI) show up as two orthogonal parts, one deterministic, one ambiguous. Singular Value Decomposition (SVD) yields the first part, optimization (usually from weighted-in, least-square data and state residuals), the second part, benefiting from a dimension of space smaller than traditional.

To further simplify the imaging procedure, weak contributors inside the ambiguous part (linked to smallest singular values of the induced-current-to-induced-current mapping operator) can be discarded, that ambiguous part then being built in a "constrained" dimension-small subspace. To run the operation in practice (since any SVD of the operator might be costly in 3-D embedding configurations), special
(Fourier) bases could be introduced, the ambiguous part being projected on those found pertinent ones (expectedly lowfrequency), e.g., [10], and applying this indeed smart approach in the present situation is under investigation, since one of the burdens of standard SOM is computational costs.

Tough questions, e.g., [11], about spectra of integral operators and choice/effect of regularizers lie beyond. Besides, in case of even weakly lossy materials, the so-called contraction integral methods that can be dated back to [12] and quite popular in geo-electromagnetics since the 2000's for modeling and imaging as well (as a recent example among many, [13]) offer us the opportunity to change the integral formulation to get a better-behaved one, now prone to simple solution expansions (Neumann-type and others), and most of it, possibly amenable to faster and more robust imaging using proper first terms of such "linearizing" expansions via transformed integral operators and field and contrast rescalings; refer to a contribution on a standard 2-D scattering case [14], the generalization/implementation of which in the 3$\mathrm{D}$ anisotropic case would be noteworthy achievement.

Support-indicator methods are also in that line of action: MUSIC-type algorithms [15-18] apply to well-separated inclusions small vs. wavelengths of probing waves, point-wise induced currents following by acting inclusion-attached polarization tensors upon incident fields; physicallyreinterpreted Sampling Methods (SM) work for large inclusions in aspect-limited (isotropic) subsurface probing, refer to pioneering [19]. The first ones (MUSIC) readily apply to a number of situations in which defects both small enough and separated enough are emerging; the second ones (SM) should enable here (as some pre-processing) to reduce the extent of the aforementioned RoI at the beginning of the study.

In brief, the purpose of these "one-shot" imaging procedures (all operating from some variant of a Multi-Static Response matrix in a source/sensor/ array configuration) is to complement the SOM-type approaches in useful fashion in terms of information effectively collected, and, since computationally low-cost, to help to systematically appraise effects of measurement configurations and data uncertainties.

Sparsity constraints have recently been proposed as effective regularizers also. Borrowing from works both general [20-21] on so-called Compressive Sensing (CS) and specialized (from ELEDIA group, as a good example among other contributors) in CS-based inverse scattering cases of electromagnetics [22-24], according to CS, the unknowns are enforced to be compressible with respect to suitable expansion bases, i.e., the corresponding vectors of expansion coefficients have few nonzero entries.

One will not dwell further on those CS (in a Bayesian framework) approaches, and concentrate upon SOM and MUSIC methods, once noticed that strong linkage between MUSIC (which implicitly relies on sparsity) and CS (that uses it in full) is investigated in [25], and that works on L1-norm minimization approaches are increasingly carried out for nonlinear inversion, possibly first via identification of supports before accessing to the material parameters themselves, e.g., for zero-frequency Electrical Impedance Tomography [26-27] and microwave imaging [28]. 


\section{ILLUSTRATIVE NUMERICAL SIMULATIONS}

The examples after are of SOM and MUSIC imaging of uniaxially anisotropic media affected by inclusions either small or not small versus the wave length. Exact datasets rely heavily on stable and efficient recurrence relations proposed to calculate the spectrum of the electromagnetic response of concerned complex materials to distributed bounded sources. From the spectral response, a fast method, including a windowing technique and a numerical integral method, follows with a fast-converging spectral asymptotic model circumventing the costly 2-D inverse Fourier transform and yielding the sought-after spatial response on a rectilinear mesh. The corresponding theoretical apparatus and many numerical investigations are in [29-30].

The above holds independently of the size of the inclusions. But, if those are small, they can be fruitfully made (or have to be made to remain accurate since the exact method is still discretization-dependent) to be equivalent within a firstorder model to point-wise fictitious sources, the amplitude of which depends on polarization tensors (accounting for anisotropic uniaxiality and expected shapes of the defects [31]) acted upon by incident fields at same location (which requires the knowledge of the dyadic Green's function between the source-receiver array and the said location). Then, MUSIC-type algorithms can be deployed if the first-order asymptotic scattered fields are close enough to the exact ones [32], RAP-MUSIC [33] being solution of choice (it was promoted in [34]) whenever several defects are expected.

Test cases are displayed next, for either three small cubical air inclusions $\left(0.1 \lambda_{1}\right.$ sided) or a somewhat large L-shaped air inclusion [35-36]. Operation frequency is $6 \mathrm{GHz}$, wavelength in air is $\lambda_{0}=5 \mathrm{~cm}$, and in the lower half-space as a uniaxial anisotropic composite with complex-valued diagonal permittivity tensor $(5.46+2.29 \mathrm{j}, 5.21+2.08 \mathrm{j}, 5.21+2.08 \mathrm{j})$, it is $\lambda_{1}=2.1 \mathrm{~cm}$ (minimal real part), with zero-degree optical axis angle. Acquisition is $0.3 \lambda_{0}$ above the interface by $13 \times 13$ electric dipoles (emitting and transmitting, with 3 orthogonal polarizations). The RoI, with top $0.25 \lambda_{1}$ below the surface, is $2 \lambda_{1} \times 2 \lambda_{1}$-sided transversely (in $x-y$ ), $0.6 \lambda_{1}$-sided in depth (z), and is sampled at $20 \times 20 \times 6$ points, accordingly.
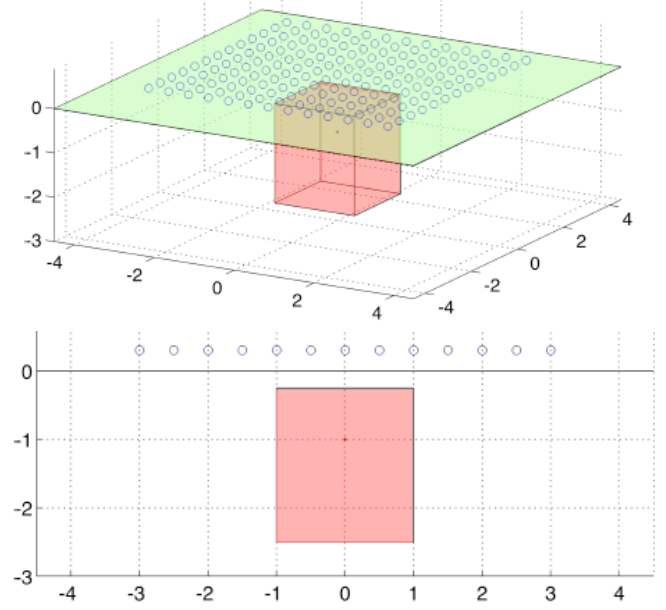

Sketch of RoI and array configuration
RAP-MUSIC iteratively yields the localization of the centers of the small defects using enhanced MUSIC [18] within the iterations. Displayed volumes are as an elementary RoI voxel. True centers are $\left[0.2 \lambda_{1}, 0.1 \lambda_{1},-0.4 \lambda_{0}\right],\left[-0.6 \lambda_{1}\right.$, $\left.0.3 \lambda_{1},-0.7 \lambda_{0}\right]$, and $\left[-0.4 \lambda_{1},-0.6 \lambda_{1},-0.9 \lambda_{0}\right]$. Data are $30 \mathrm{~dB}$ noisy (left side of the image) and $10 \mathrm{~dB}$ noisy (right side).

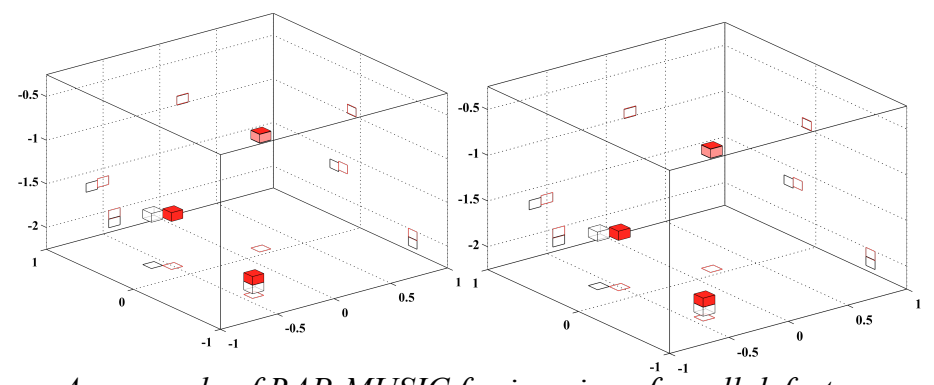

An example of RAP-MUSIC for imaging of small defects

SOM is on the retrieval of the L-shaped object (displayed first, 3D and projection into $x-y$ plane). It extends (in $z$ ) on layers 4 and 5. The $x x$ dielectric contrast component of (due to anisotropy, 6 unknown complex-valued components) is next, ordered clockwise layers 1 to 6 , real parts (two upper rows) and imaginary ones (two lower rows). Similar results hold for $y y$ and $z z$ components. Data are noiseless, but noise does not change the results much.
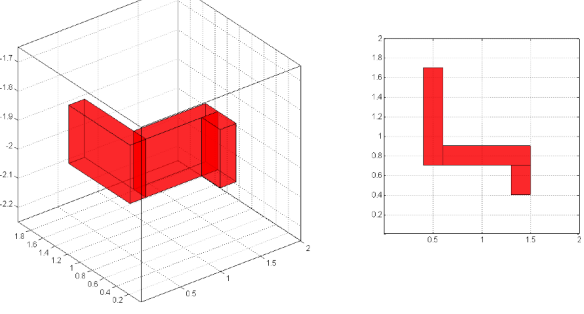

An example of SOM for imaging of a large defect
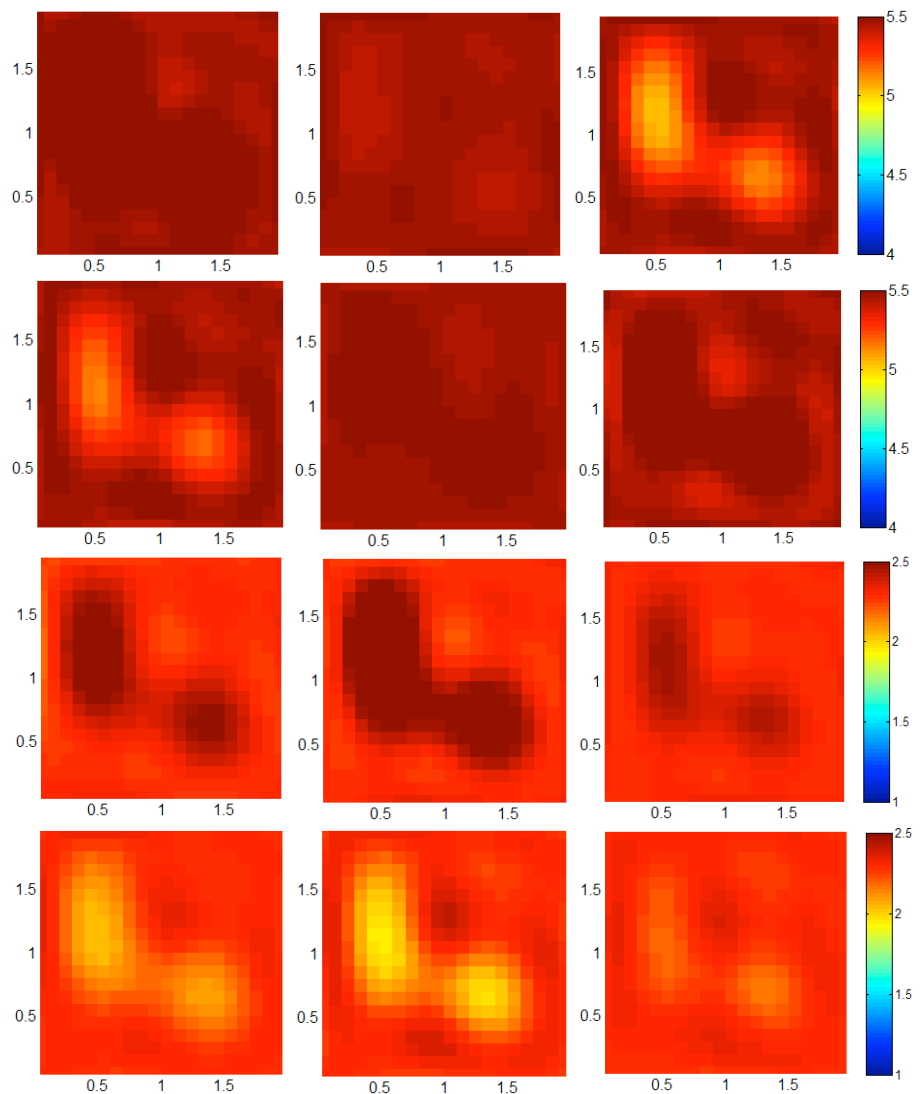


\section{References}

[1] S. Kharkovsky and R. Zoughi, "Microwave and millimeter wave nondestructive testing and evaluation. Overview and recent advances," IEEE Instrum. Measur. Mag., Vol. 10, pp. 26-38, 2007.

[2] H. Heuer et al., "Review on quality assurance along the CFRP value chain - Nondestructive testing of fabrics, preforms and CFRP by HF radio wave techniques," Composites part B, Vol. 77, pp. 494-501, 2015.

[3] C. Y. Li, D. Lesselier, and Y. Zhong, "Recursive matrix schemes for multilayered composite with periodically arranged fibers under plane wave or Gaussian beam illumination”, J. Optical Soc. Amer. B, Vol. 32, pp. 1539-1549, 2015.

[4] A. S. Bin Sediq and N. Qaddoumi, "Near-field microwave image formation of defective composites using open-ended waveguides with arbitrary cross sections," Composite Struct., Vol. 71, pp. 343-348 2005.

[5] Y.-H. Teo, X. Wang, and W.-K. Chiu, "Simulations of microwave propagation in delaminated unidirectional glass-epoxy laminate," Composite Struct., Vol. 75, pp. 422-427, 2006.

[6] C. Stoik, M. Bohn, and J. Blackshire, "Nondestructive evaluation of aircraft composites using reflective TeraHertz time domain spectroscopy," NDT\&E Intern., Vol. 43, pp. 106-115, 2010.

[7] Y. Zhong and X. Chen, "Twofold subspace-based optimization method for solving inverse scattering problems," Inverse Probl., Vol. 25, 085003 (11pp), 2009.

[8] K. Agarwal, L. Pan, and X. Chen, "Subspace-based optimization method for reconstruction of 2-D complex anisotropic dielectric objects," IEEE Trans. Microw. Theory Techn, Vol. 58, pp. 1065-1074, 2010.

[9] G. Oliveri, Y. Zhong, X. Chen, and A. Massa, "Multi-resolution subspace-based optimization method for inverse scattering problems," J. Optical Soc. Amer. A, Vol. 28, pp. 2057-2069, 2011.

[10] Y. Zhong and X. Chen, "An FFT twofold subspace-based optimization method for solving electromagnetic inverse scattering problems," IEEE Trans. Antennas Propagat., Vol. 59, pp. 914-927, 2011.

[11] N. V. Budko and A. B. Samokhin, "Spectrum of the volume integral operator of electromagnetic scattering," SIAM J. Scientific Comp., Vol. 28 , pp. 682-700, 2006

[12] O. V. Pankratov, A. V. Kuvshinov, and D. B. Avdeev, "Highperformance three-dimensional electromagnetic modelling using modified Neumann series. Anisotropic Earth," J. Geo-magn. Geo-elec., Vol. 49, pp. 1541-1547, 1997.

[13] L. H. Cox and M. S. Zhdanov, "Advanced computational methods of rapid and rigorous 3-D inversion of airborne electromagnetic data," Communic. Computation. Phys., Vol. 3, pp. 160-179, 2008.

[14] Y. Zhong, M. Lambert, D. Lesselier, and X. Chen, "A new integral equation method to solve highly nonlinear inverse scattering problems," submitted to IEEE Trans. Antennas Propagat., 2015.

[15] H. Ammari, E. Iakovleva, D. Lesselier, and G. Perrusson, "MUSIC-type electromagnetic imaging of a collection of small 3-D bounded scatterers," SIAM J. Scientific Comput., Vol. 29, pp. 674-709, 2007.

[16] E. Iakovleva and D. Lesselier, "Multi-Static Response matrix of spherical scatterers and the back-propagation of singular fields," IEEE Trans. Antennas Propagat., Vol. 56, pp. 825-833, 2008.

[17] H. Ammari, P. Calmon, and E. Iakovleva, "Direct elastic imaging of a small inclusion,” SIAM J. Imag. Sci., Vol. 1, pp. 169-187, 2009.

[18] X. Chen and Y. Zhong, "MUSIC electromagnetic imaging with enhanced resolution for smal inclusions," Inverse Probl., Vol. 25, ID:015008 (12pp), 2009.

[19] I. Catapano, L. Crocco, and T. Isernia, "Improved sampling methods for shape reconstruction of 3-D buried targets," IEEE Trans. Geosci. Remote Sens., Vol. 46, pp. 3265-3273, 2008.
[20] E. J. Candes and M. B. Wakin, "An introduction to compressive sampling," IEEE Signal Process. Mag., Vol. 25, pp. 21-30, 2008.

[21] J. A. Tropp and S. J. Wright, "Computational methods for sparse solution of linear inverse problems," Proc. IEEE, Vol. 98, pp. 948-958, 2010.

[22] L. Poli, G. Oliveri, and A. Massa, "Microwave imaging within the firstorder Born approximation by means of the contrast-field Bayesian compressive sensing," IEEE Trans. Antennas Propagat., Vol. 60, pp. 2865-2879, 2011.

[23] L. Poli, G. Oliveri, P. Rocca, and A. Massa, "Bayesian compressive sensing approaches for the reconstruction of two-dimensional sparse scatterers under TE illuminations," IEEE Trans. Geosci. Remote Sens., Vol. 51, pp. 2920-2936, 2013.

[24] L. Poli, G. Oliveri, P.-P. Ding, T. Moriyama, and A. Massa, "Multifrequency Bayesian compressive sensing methods for microwave imaging," J. Optical Soc. Amer. A, Vol. 31, pp. 2415-2428, 2014.

[25] A. C. Fannjiang, "The MUSIC algorithm for sparse objects: a compressed sensing analysis," Inverse Probl., Vol. 27, 035013 (32pp), 2011.

[26] B. Jin, T. Khan, and P. Maass, "A reconstruction algorithm for electrical impedance tomography based on sparsity regularization," Int. J. Numer. Meth. Engng., Vol. 89, pp. 337-353, 2012.

[27] O. K. Lee, H. Kang, J. C. Ye, and M. Lim, "Non-iterative method for the electrical impedance tomography based on joint sparse recovery," Inverse Probl., Vol. 31, 075002 (23pp), 2015.

[28] A. Desmal and H. Bağcı, "Shrinkage-thresholding enhanced Born iterative method for solving 2D inverse electromagnetic scattering problem," IEEE Trans. Antennas Propagat., Vol. 62, pp. 3878-3883, 2014.

[29] Y. Zhong, M. Lambert, D. Lesselier, and X. Chen, "Electromagnetic response of anisotropic laminates to distributed sources," IEEE Trans. Antennas Propagat., Vol. 62, pp. 247-256, 2014.

[30] Y. Zhong, P. Ding, M. Lambert, D. Lesselier, and X. Chen, "Fast calculation of scattering by 3-D inhomogeneities in uniaxial anisotropic multilayers," IEEE Trans. Antennas Propagat., Vol. 62, pp. 6365-6374, 2014.

[31] W. S. Weiglhofer and A. Lakhtakia, "New expressions for depolarization dyadics in uniaxial dielectric-magnetic media," Intern. J. Infrared Millim. Waves, Vol. 17, pp. 1365-1367, 1996.

[32] G. Rodeghiero, P.-P. Ding, Y. Zhong, M. Lambert, D. Lesselier, MUSIC imaging method for low-high frequency inspection of composite multilayers, 41st Rev. Progr. Quantit. Nondestr. Eval., AIP Conf. Proc. 1650 L. J. Bond and D. E. Chimenti eds., pp. 453-461, New York, 2015.

[33] J. C. Mosher and R. M. Leahy, "Source localization using recursively applied and projected (RAP) MUSIC,” IEEE Trans. Sign. Process., Vol. 47, pp. 332-340, 1999.

[34] E. Iakovleva and D. Lesselier, "On the MUSIC-type electromagnetic imaging of a small collection of dielectric spheres from its multi-static response matrix using exact and asymptotic numerical data," 23rd Annual Rev. Progr. Appl. Computation. Electromagn.,Verona, March 2007 (available from http://www.aces-society.org/).

[35] P.-P. Ding, M. Lambert, and D. Lesselier, "Subspace-based optimization method for reconstructing 3-D scatterers in anisotropic laminates', 20th Int. Workshop Electromagn. Non-Destr. Eval., ENDE 2015, Sendai, Sept. 2015.

[36] P.-P. Ding, G. Oliveri, L. Poli, and A. Massa, "Reconstruction of 3D objects in anisotropic multi-layered media by a Bayesian compressive sensing solver," 17th Intern. Symp. Applied Electromagn. Mechan., ISEM 2015, Kobé, Sept. 2015 